\title{
Entrüstigter Rentner
}

\author{
RED FLAGS ERKENNEN Eine harte Nuss: Herr Kanter klagt über diffuse Schmerzen \\ im Kniegelenk, Hüftgelenk und im Rücken. Physiotherapeut Dr. Harry von Piekartz kann \\ diese selbst nach einer ausgiebigen Untersuchung nicht einordnen. Erst ein Internist \\ findet die Ursache. Sie ist lebensgefährlich.
} im Ruhestand ist. Seit acht Wochen plagen ihn Schmerzen im rechten Knie, die sich über den Oberschenkel in Richtung Hüfte und Bauch ausbreiten ( $\odot \mathbf{A b b}$. 1). Er hat auch das Gefühl, sein rechtes Bein nicht richtig kontrollieren zu können. Seine Beschwerden schränken Peter Kanter stark ein: Spazieren gehen und im Garten arbeiten kann er nicht mehr, und auch sein anderes Hobby - Tanzen - bereitet ihm starke Beschwerden. Die Schmerzen werden im Stehen schlimmer, Gehen beeinflusst die Symptome nicht. Morgens, wenn Herr Kanter aufsteht, beginnen die Beschwerden direkt und haben eine Stärke von 3 auf einer 10-Punkte-visuellen Analogskala (VAS). Tagsüber werden sie schlimmer (VAS 7/10). Wenn er sich auf seine rechte Seite legt, geht es ihm oft nach einer halben Stunde besser (VAS 3/10). Steht er danach auf, nehmen die Schmerzen nach zehn Minuten wieder zu. Nachts wird er davon durchschnittlich fünf Mal wach. Er hat bemerkt, dass er dann auf dem Rücken liegt. Dreht er sich auf die rechte Seite, schläft er wieder ein.

Begonnen hat das Problem vor zehn Wochen, und zwar mit diffusen Beschwerden im thorakolumbalen Übergang. Damals hatte Peter Kanter viel in seinem Garten gearbeitet und Holz gesägt, was seiner Meinung nach die Schmerzen ausgelöst haben könnte. Besser werden die Rückenschmerzen in LWS-Flexion, zum Beispiel wenn er sitzt oder sich im Stehen auf einem Tisch abstützt. Massage verschlechterte die Beschwerden. Seit ihm Knie und Hüfte weh tun, hat er zwar das Gefühl, dass der Rücken besser würde. Insgesamt verschlechtern sich die Beschwerden jedoch.

Rückenschmerzen hatte Peter Kanter schon einmal, damals jedoch eher im unteren LWS-Bereich. Aktuell hat der Hausarzt das Abdomen und die LWS klinisch untersucht - ohne Befund. Auf Röntgenbildern von LWS, Hüft- und Kniegelenk sieht man, dass diese Bereiche altersentsprechend degeneriert sind. Als Nebendiagnose hat Herr Kanter seit 13 Jahren eine chronische arterielle Hypertonie, er raucht, seit er 18 ist, und mit 35 wurde er an einem Leistenbruch operiert. Sein Gesundheitszustand ist insgesamt gut.

Dass sich Peter Kanters Symptome verbessern, wenn er seine Wirbelsäule flektiert, und sich verschlechtern, wenn er geht, passt nicht zu einem diskogenen LWS-Problem, eher zu einer degenerativen LWS-Dysfunktion wie einer lumbalen Spinalkanalstenose (๑ „Lumbale Spinalkanalstenose“). Mich irritiert jedoch, dass keine entsprechenden Veränderungen auf dem Röntgenbild zu sehen sind. Die würde ich erwarten - vor allem bei einer solch progressiven Vorgeschichte und derart deutlichen unilateralen Beschwerden. Trotzdem werde ich noch einmal genau bezüglich einer spinalen Stenose nachfragen. 


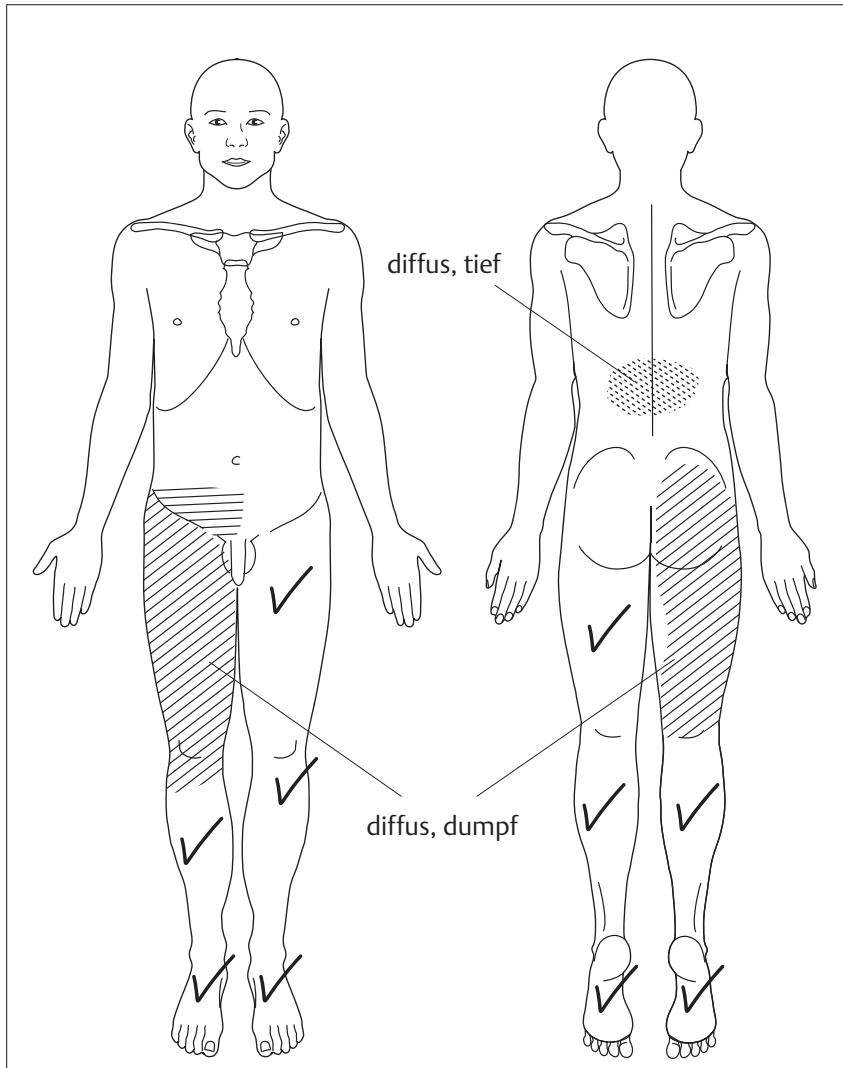

Abb. 1 Diffuse Beschwerden: Peter Kanter hat Schmerzen im rechten Knie und Oberschenkel, der Hüfte sowie im Bauch. Der erste Verdacht „Spinalkanalstenose“ bestätigt sich nicht.
Bei Herrn Kanter zeigen sich zuerst die Schmerzen im Kniegelenk und danach die Beschwerden im Hüftgelenk. Zudem treten die Schmerzen nicht nur auf, wenn er geht, sondern auch, wenn er auf dem Rücken liegt. Ich frage noch einmal nach, ob die Schmerzen tatsächlich plötzlich begonnen haben oder eher schleichend. Peter Kanter beharrt darauf, dass die Beinschmerzen plötzlich begonnen haben, nachdem er zuvor zwei Wochen lang unter Rückenschmerzen gelitten hatte.

In der Regel sind die Symptome bei einer lumbalen Stenose diffus und gehen durch das ganze Bein. Sie treten nicht auf, wenn der Patient auf dem Rücken liegt, beginnen langsam und steigern sich über Wochen und Monate. Somit ist es fraglich, ob Herrn Kanters Symptome tatsächlich durch eine lumbale Spinalkanalstenose ausgelöst werden. Da die Symptome einseitig sind, könnten sie auch zu einer Koxarhtrose passen oder einer peripheren Neuropathie des N. femoralis.

Eine Koxarthrose kann mit starken Kniegelenkschmerzen beginnen und sich verschlechtern, wenn der Patient steht oder geht. Doch Herr Kanter klagt weder über eine Steifigkeit im rechten Hüftgelenk, noch hat er einen deutlich asymmetrischen radiologischen Befund. Auch dass das Liegen auf der linken Seite die Symptome lindert, ist für eine Koxarthrose untypisch.

Eine Neuropathie des $N$. femoralis verursacht Schmerzen und kann zu einem Kontrollverlust im Bein führen. Peter Kanters Symptome passen zum Versorgungsgebiet des N. femoralis, und seine thorakolumbalen Beschwerden sind in dem Bereich lokalisiert, in dem die Nervenwurzel des N. femoralis entspringt (L 1-L3). Dass er in Rückenlage Schmerzen bekommt, könnte mit dem alten Leistenbruch und einer dadurch eingeschränkten Neurodynamik des N. femoralis zusammenhängen. Was dagegen eher nicht passt, ist die Qualität der Knie- und Hüftgelenkschmerzen: Bei einer Neuropathie würde man erwarten, dass sie einschießen und sich eher von proximal nach distal ausbreiten, nicht andersherum.

\section{LUMBALE SPINALKANALSTENOSE}

\section{Klinische Zeichen einer Stenose}

$>$ Männer häufiger betroffen als Frauen

$>$ Auftreten zwischen dem 50.-70. Lebensjahr

$>$ beginnt in der Regel ohne klaren Auslöser

$>$ allmählich verschlechternde Symptomatik

$>$ eher diffuse Rückenschmerzen

$>$ selten monoradikuläres Syndrom, eher diffuse Beinschmerzen

$>$ häufig „restless leg syndrome“ während der Nacht

$>$ Symptomzunahme bei LWS-Extension (z. B. Gehen)

$>$ Symptomabnahme bei LWS-Flexion (z. B. Vorneigen)

$>$ Husten und Niesen ändert Symptome nicht

\section{DIFFERENZIALDIAGNOSTIK}

\section{Klinische Zeichen einer arteriellen Verschlusskrankheit}

\section{Einengung der Bifurcatio aortae:}

$>$ muskulärer Funktionsverlust und Atrophie, vor allem des M. iliopsoas

$>$ nicht segmentale Taubheit

$>$ Parästhesien (Brennen und Kribbeln)

$>$ Lähmungen

$>$ Claudicatio intermittens

$>$ Schmerzen in Rücken, Hüftgelenk und Beinen

$>$ kalte, zyanotische Beine

$>$ verringerter peripherer Puls

\section{Einengung der Arteria iliaca:}

$>$ Claudicatio intermittens

$>$ Schmerzen in der Region Hüftgelenk/ Taille

$>$ Impotenz bei Männern

$>$ verringerter arterieller Puls 


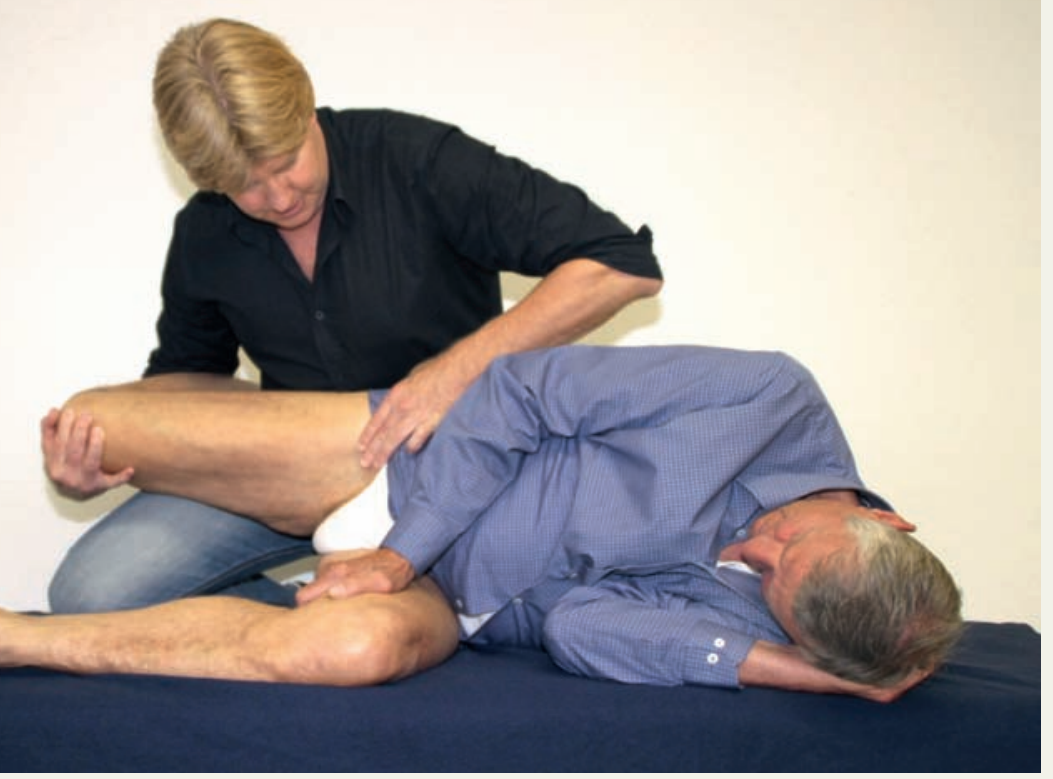

Abb. 2 Prone Knee Bend in Seitenlage: Ein zusätzlicher, leichter Druck auf die A. femoralis verstärken Herrn Kanters Schmerzen im Bein und die diffusen Schmerzen im Bauch. Das könnte auf eine arterielle Verschlusskrankheit hindeuten.

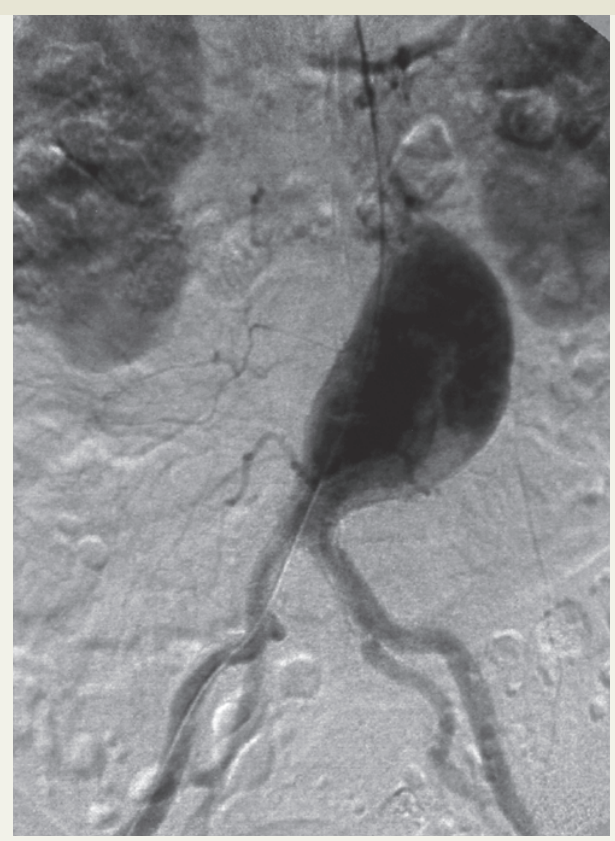

Abb. 3 Infrarenales Aortenaneurysma: Die Angiografie ist das Mittel der Wahl, um eine solche Aussackung zu erkennen.
Untersuchung des Kniegelenks und der LWS:

> Inspektion im Stand: Leichte Flexion/Kyphosierung der LWS, rechtes Bein etwas im Hüftgelenk nach außen rotiert. Bei der Korrektur tritt ein leichter Druck in der rechten Leiste auf.

$>$ Untersuchung des Kniegelenks rechts: physiologische Bewegungen o. B.; Zusatzbewegungen, Kompression und Distraktion von Tibio- und Patellofemoralgelenk o. B.

> Aktives Bewegungsausmaß der LWS: Extension, Flexion, Lateralflexion und Rotation sind beidseits altersentsprechend reduziert, aber ohne Beschwerden.

\section{Neurologische Untersuchung der unteren Extremität:}

> Sensibilität: Schlecht abgrenzbare Hyperalgesie im Bereich des ventralen und lateralen Oberschenkels

> Kennmuskulatur (manuelle Widerstandstests): Kniegelenkextension (Quadrizeps / L3) o. B., Hüftgelenkflexion (Iliopsoas / L2) sehr schmerzhaft, Kraft verringert

$>$ Zentral neurologische Tests: Fußsohlenreflex („Babinski“) o. B.

$>$ Neurodynamische Untersuchung:

> Straight Leg Raise (SLR, „Lasègue“): Bei zusätzlicher Adduktion im rechten Hüftgelenk bemerkt Peter Kanter rechts einen „Druck in der Leiste“. Außerdem bekommt er „diffuse Bauchschmerzen“.

> Prone Knee Bend (PKB, Beugen des Kniegelenks) in Bauchlage: Links löst der Test bei $130^{\circ}$ Kniegelenkflexion ein „Ziehen im Oberschenkel“ aus; rechts bekommt Herr Kanter bei $40^{\circ}$ Kniegelenkflexion nach ein paar Sekunden Bauchschmerzen und anschließend seine bekannten Schmerzen im Kniegelenk. Nackenflexion beeinflusst die Symptome nicht.
> PKB in Seitenlage (Vorpositionierung des Oberkörpers im „Slump“): gleiches Ergebnis wie in Bauchlage

> Slump-Test im Sitz: o. B.

Untersuchung des Hüftgelenks: > Alle physiologischen Bewegungen sind seitengleich unauffällig. Lediglich bei Adduktion in $90^{\circ}$ Flexion tritt rechts in gehaltener Position ein lokaler Schmerz auf, der anschließend noch über 20 Sekunden anhält. Kompression und Distraktion des Hüftgelenks sind unauffällig.

Herr Kanters Symptome sind untypisch für eine neuromuskuloskeletale Störungen von Kniegelenk, Hüftgelenk und LWS. Nur der PKB in Bauchund Seitenlage reproduziert die Schmerzen. Sensibilisierende Bewegungen, die das Nervensystem zusätzlich auf Spannung bringen - zum Beispiel die Nackenflexion -, sind negativ. Möglicherweise irritiert die intraabdominale Narbe der Blinddarmoperation Herrn Kanters rechten N. femoralis?

Ich palpiere die Blinddarmnarbe am Unterbauch. Sie ist nicht schmerzhaft, und ich finde keine Adhäsionen. Um auch einen Eindruck von den anderen Strukturen zu bekommen, palpiere ich danach routinemäßig die Lymphknoten und die Arteria femoralis.

Die Palpation der Lymphknoten ist ebenfalls unauffällig. Als ich jedoch den Puls an der A. femoralis taste, bemerke ich, dass er in Rückenlage zwar links deutlich zu spüren ist, rechts dagegen gar nicht $(F)$. Auch als Herr Kanter auf der linken Seite liegt, ist der Pulsschlag auf der rechten Seite gegenüber links um rund $50 \%$ vermindert $(F)$. Ich teste den PKB in Seitenlage noch einmal und drücke dabei leicht auf die A. femoralis. Nach 20 Sekunden beginnen die Beinschmerzen und die diffusen Bauchschmerzen ( $\odot \mathbf{A b b} .2$ ). 
Aufgrund von Peter Kanters Alter, seiner beitragenden Faktoren (Hypertonie, langjähriges Rauchen) und den klinischen Symptomen muss ich nun in Erwägung ziehen, dass seine Beschwerden nicht von einem muskuloskeletalen Problem, sondern einer arteriellen Verschlusskrankheit herrühren könnten (๑ „Differenzialdiagnostik“, S. 23).

Ich entschließe mich, Herrn Kanter nicht zu behandeln. Stattdessen rate ich ihm, sich weiter medizinisch abklären zu lassen. Ich gebe ihm meine Befunde mit und informiere seinen Hausarzt telefonisch. Dieser überweist ihn weiter zum Internisten, der eine Ultraschalluntersuchung des Bauchraums veranlasst sowie eine Computertomografie und eine Angiografie. Dabei findet man ein sechs Zentimeter großes Aortenaneurysma. Es sitzt infrarenal, also unterhalb des Abgangs der Nierenarterien ( $\odot \mathbf{A b b}$. 3). Diese vaskuläre Aussackung hat möglicherweise den N. femoralis leicht komprimiert, der in einer geschlossenen Faszie auf dem M. iliopsoas direkt neben der Aorta liegt, und damit die Beschwerden verursacht. Herr Kanter unterzieht sich einer OP, in der das Aneurysma entfernt wird.

Drei Monate später stellt er sich erneut bei mir vor. Seine Beschwerden sind komplett verschwunden, und sämtliche Aktivitäten kann er wieder uneingeschränkt ausführen.

Harry von Piekartz

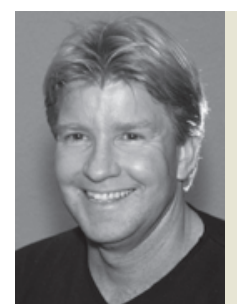

Prof. Dr. Harry von Piekartz ist Professor für Physiotherapie an der Hochschule Osnabrück, Präsident der Cranio Facial Therapy Academy (Crafta) sowie Fachlehrer für das Neuro-orthopädische Institut (NOI) und das Maitland-Konzept (IMTA). In dieser Artikelserie beschreibt er Fälle aus der eigenen Praxis.

\section{Aortenaneurysma}

Bei Aneurysmata handelt es sich um Aussackungen der Arterien. Grundsätzlich gibt es zwei unterschiedliche Arten: angeborene und erworbene. Die angeborenen bleiben häufig über lange Zeit symptomlos - bis sie dann ohne Vorwarnung rupturieren. Lokalisiert sind sie fast immer in der Hirnstrombahn, vor allem in den Hirnbasisgefäßen. Reißt ein solches Aneurysma, kommt es zu einer intrakraniellen subarachnoidalen Blutung. Ihr Leitsymptom: „Kopfschmerz wie noch nie“. Im Gegensatz zu Schlaganfällen - denen zu über 85 \% thrombotische Verschlüsse zugrunde liegen und eben keine Blutung - sind die von einem Hirnarterien-Aneurysma betroffenen Patienten deutlich jünger (Altergipfel: 40.-60. Lebensjahr). Die Prävalenz in Deutschland beträgt etwa $1 \%$. Pro Jahr erleiden rund 10 von 100.000 Einwohnern aufgrund eines Aneurysmas eine Hirnblutung.

Herr Kanter hatte sehr wahrscheinlich eine erworbene Variante in der Aorta. $80 \%$ der dort auftretenden Aneurysmata sitzen im abdominalen Bereich, $20 \%$ im thorakalen (๑ Abb. 4, S. 26). Auslöser sind sämtliche Risikofaktoren der Arterienverkalkung: neben Bewegungsmangel insbesondere inhalatives Rauchen, Fettstoffwechselstörungen und arterielle Hypertonie. Männer sind deutlich häufiger betroffen als

Frauen. Auch Patienten mit dem MarfanSyndrom, einer Kollagenosestörung mit typischem äußerem Erscheinungsbild (u. a. schlanker Hochwuchs, Spinnenfingrigkeit und überstreckbare Gelenke), leiden häufig an thorakalen Aneurysmata.

\section{Höchste Rupturgefahr: Aneurysma dissecans}

Es gibt verschiedene Arten von Aneurysmata (॰ Abb. 5, S. 26). Sacken alle drei Teile der arteriellen Gefäßwand (Intima, Media und Adventitia) gleichermaßen aus, liegt ein Aneurysma verum vor. Kommt es zwischen der inneren Gefäßauskleidung (Intima) und der stark mit glatten Muskelzellen ausgestatteten Media zu einem Riss, spricht man von einem Aneurysma dissecans. Ein solcher Riss füllt sich mit Blut, das dann gerinnt. Dieser Prozess setzt sich unter Umständen immer weiter fort. Dadurch entsteht neben dem echten Gefäßlumen, durch das das Blut weiterhin fließt, auch ein „falsches“. Die Rupturgefahr ist beim Aneurysma dissecans besonders groß. Auch nach arteriellen Gefäßpunktionen, beispielsweise der Anlage von Herzkathetern (durch die Leistenarterie oder eine
Armarterie), kann eine Gefäßaussackung entstehen - das Aneurysma spurium.

Das abdominale Aortenaneurysma liegt - wie auch im Falle von Herrn Kanter - in 95\% der Fälle infrarenal, also unterhalb der Abgänge der Nierenarterien (Aa. renales). Daraus resultieren die typischen Symptome, vor allem die nach dorsal ausstrahlenden Schmerzen und die Kompression des N. femoralis. Andere mögliche, meist lageabhängige Symptome sind Thoraxschmerzen, Schluckstörungen und Obstipation. Bei der lebensbedrohlichen Ruptur bekommt der Patient plötzlich

\section{Männer sind deutlich häufiger von Aneurysmata betroffen als Frauen.}

Dauerschmerzen und einen Schock. Auch in der Peripherie treten Aneurysmata auf, vorwiegend in $\operatorname{der}$ A. poplitea, der A. femoralis communis, der A. iliaca und der A. subclavia. Dort kann man sie als sich deutlich ausdehnende, pulsierende Tumore tasten. Aussackungen in der Peripherie reißen allerdings eher selten. 


\section{Diagnostik: Angiografie, Therapie: Angioplastie}

Das erste Symptom eines abdominalen Aneurysmas sind Rückenschmerzen, je nach Lage der Aussackung in der thorakalen oder lumbalen Wirbelsäule. Große sogenannte Bauchaortenaneurysmata sind als prall-elastischer, pulsierender Tumor im Abdomen tastbar und auskultierbar. Bildgebende Verfahren wie Sonografie, CT, MRT und Angiografie sichern die Verdachtsdiagnose.

Für die Praxis gilt: Bei Rückenschmerzen, die vom Abdomen oder Becken fortgeleitet werden, aber ohne wesentliche Bewegungseinschränkungen bzw. ohne Bewegungsschmerz der Wirbelsäule auftreten, sollte man hellhörig werden. Ob physiotherapeutische Techniken, beispiels-

\section{Zunächst ist wichtig, die Risikofaktoren zu managen, allen voran die Hypertonie.}

weise spinale Manipulationen („High-velocity-low-amplitude-thrust-Techniken“) eine ungewollte Aneurysma-Ruptur auslösen können, ist zwar nicht ausreichend geklärt - trotzdem ist hier größte Vorsicht geboten. Jeglicher Verdacht auf ein Aneurysma muss umgehend ärztlich abgeklärt werden.

In der Therapie muss man zunächst die Risikofaktoren managen, allen voran die Hypertonie. Daneben besteht die Möglichkeit, in einem offenen gefäßchirurgischen Eingriff eine Prothese in die betroffene Arterie einzusetzen. Auch endoskopische Verfahren kommen zunehmend zum Einsatz, bei denen mittels Katheter, ähnlich wie bei den Herzkranzgefäßen (Koronarangioplastie), ein sogenannter Stent in das betroffene Gefäßgebiet implantiert wird. Christoff Zalpour
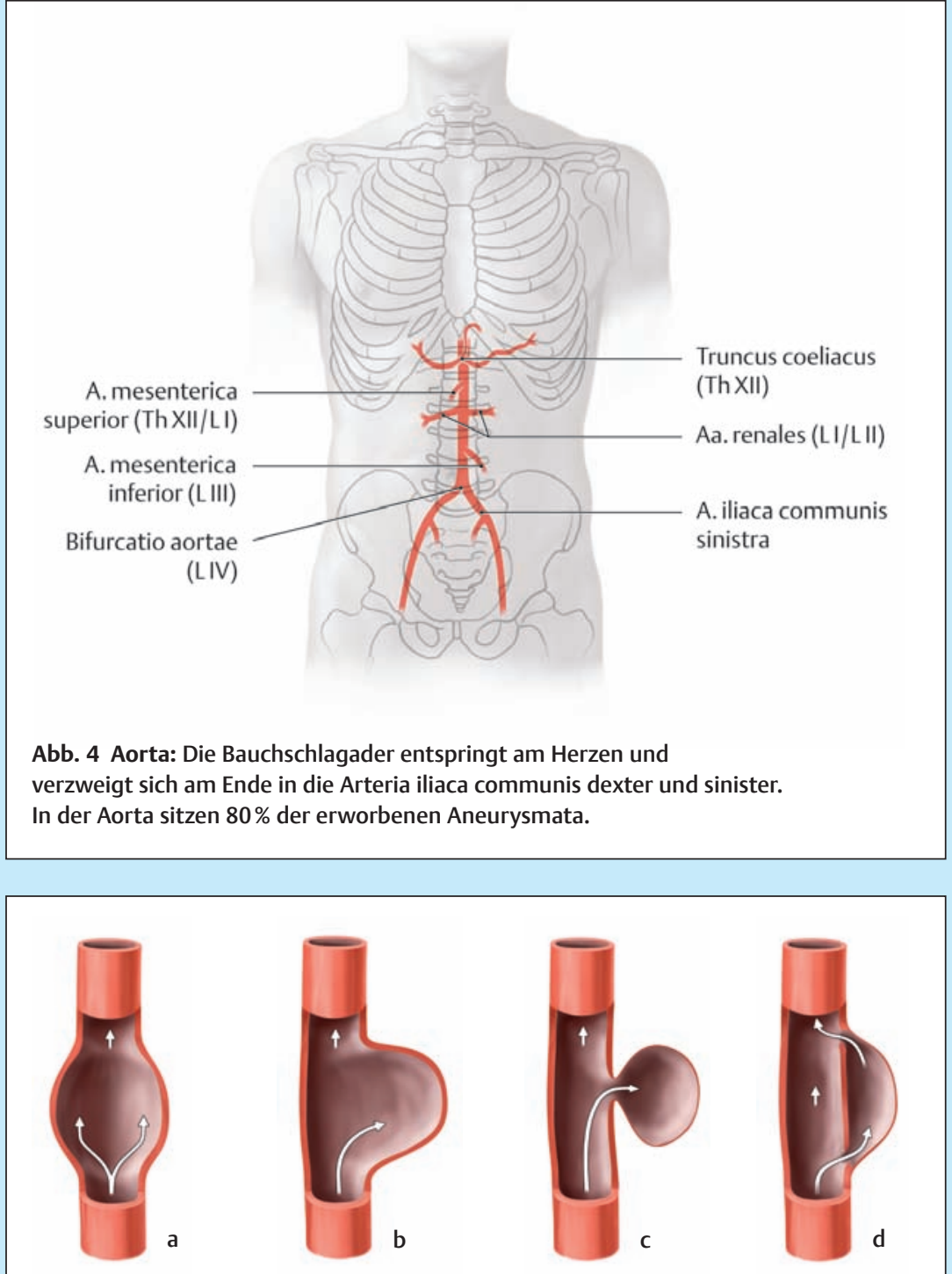

Abb. 5 Arten des Aneurysmas: Ein Aneurysma kann gleichförmig ausgedehnt sein (a) oder einseitig (b). Ein Aneurysma spurium (c) entsteht beispielsweise nach einer Punktion. Bei einem Aneurysma dissecans (d) strömt das Blut durch zwei Lumen. Hier ist die Rupturgefahr am größten.

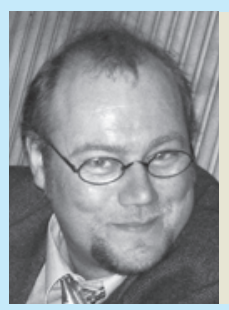

Prof. Dr. Christoff Zalpour ist Mediziner und Professor für Physiotherapie an der Hochschule Osnabrück. Dort leitet er zudem das Institut für angewandte Physiotherapie und Osteopathie und ist Sprecher der AG SCIPOS (Science in Physiotherapy in Osnabrück). 